\title{
Structural Analysis of 3D Printing Model
}

\author{
Chengcheng $\mathrm{Li}^{1, \mathrm{a}}{ }^{*}$ and Jinxian $\mathrm{Qi}^{2, \mathrm{~b}}$ \\ ${ }^{1}$ Key Laboratory of Metallurgical Equipment and Control Technology, Ministry of Education, \\ Wuhan University of Science and Technology, Wuhan 430081, China. \\ ${ }^{2}$ Hubei Key Laboratory of Mechanical Transmission and Manufacturing Engineering, \\ Wuhan University of Science and Technology, Wuhan 430081, China. \\ a1271693864@qq.com, b1847820924@qq.com
}

Keywords: Printing technology; Model optimization; Structural analysis

\begin{abstract}
D printing is a rapid prototyping technology which became popular in the last two years. It builds objects through layer-by-layer printing, which is based on digital model files. As a new technology in rapid manufacturing, 3D printing is rapidly emerging and will become an important part of the manufacturing industry in the future. This paper introduces the basic principle of 3D printing technology and the current development trend. The printing model is analyzed in detail from four aspects of saving printing materials, improving the strength, increasing the stability and improving the supporting structure, and the related optimization methods are put forward. Structural optimization can reduce the consumption of printed materials, reduce printing costs and eliminate the structural defects of the 3D model. Through the optimization of 3D model, 3D printing technology can continue to make progress.
\end{abstract}

\section{Introduction}

$3 \mathrm{D}$ printing is a rapid prototyping technology based on mathematic model files that create objects from bottom to top by layering materials such as ceramics, plastics and powdered metals. Compared with the traditional "subtractive manufacturing" of machining, 3D printing is an additive manufacturing technology, which is a manufacturing technology that is realized by the principle of discrete and dimensional product. 3D printing has many different core technologies that print complete 3D models layer by layer with different materials. 3D printing technology directly combines design theory with process manufacturing, It can print product quickly. From the model to the product, It can facilitate the development of innovative thinking and spot problems and make improvements in the printing process. As a result, more and more attention has been paid in many fields such as mechanical design and manufacturing, aerospace, architectural design, art design and biomedicine. In order to promote the development of 3D printing, we need to analyze the structure of the 3D printed model, the materials used in the model, the strength between the various parts of the model and the stability of the model. So that the model produced can be well applied to practice to meet the actual processing requirements and reduce the corresponding production costs and time costs.

\section{The principle and advantage of 3D printing technology}

The working principle of the 3D printer is actually similar to the principle of an ordinary daily printer. The daily life printer is a two-dimensional planar object, and the 3D printer first draws a virtual 3D model on the computer, and then the virtual 3D model is cut into a layer of cross section, which is slicing process, and finally guide the 3D printer from bottom to top layer by layer printing. In general, as shown in figure 1, the process of 3D printing is mainly divided into four areas, namely: building models, slicing, printing, post-processing.

To Build a Model. To print the actual model product, it is necessary to draw a corresponding three-dimensional virtual model. Therefore, we must first use the three-dimensional graphics 
software (such as CAD, Pro/E, etc.)to design the appropriate software model, according to our own ideas.

Layered Slices. The design of the 3D virtual model input to the computer to go, and then through the 3D printer comes with professional printing software to further print processing, the model cut into layers of sheet, the properties of the print material and the printer's specifications decided the thickness of each sheet.

Printing and Forming. 3D printer model printing process is mainly divided into two steps: support the production and the production of parts entities. In the printed area, the printer head travels along the path specified by the programming software to extrude the liquid and support materials and print the model up from the bottom up.

Post-processing. The post-processing of 3D printing technology includes: stripping excess support material from the model while ensuring the shape of the model surface, preventing large deformation of the model, and processing the surface to meet the accuracy requirements of the model.

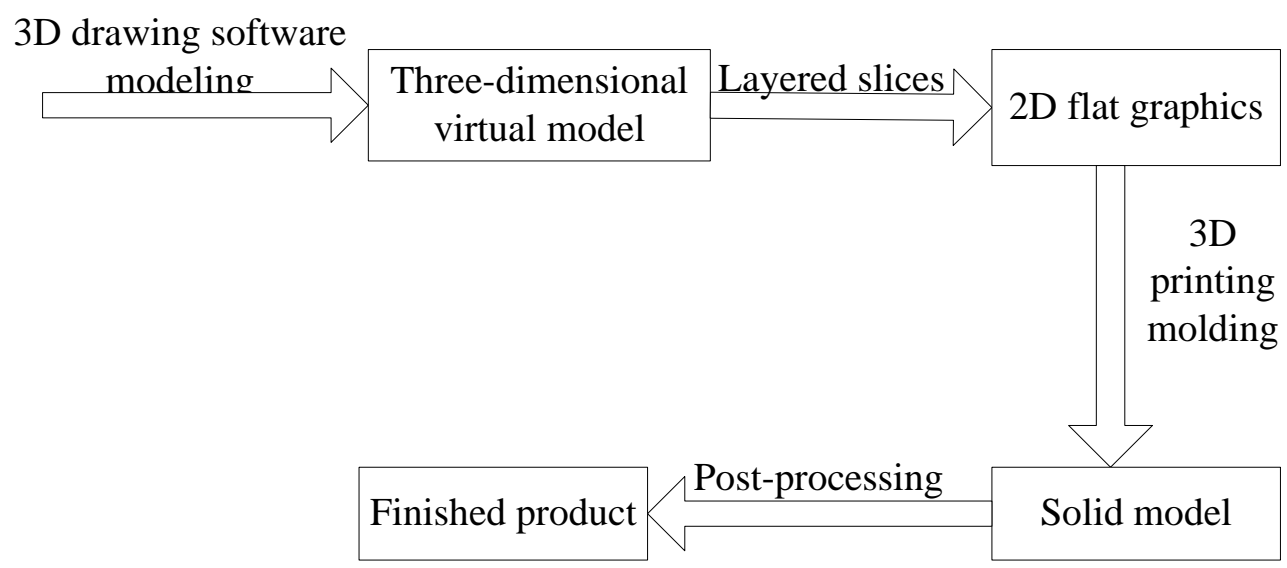

Figure 1. 3D printing process flow chart

3D printing combines the design of the model with the production of the process, and the steps that were previously required to be done separately can now be made in $3 \mathrm{D}$ by all means. This greatly reduces the product model in the design of production costs, but also significantly shorten the production time[1].3D printing does not require the same as the traditional processing in a variety of workshops gradually precision machining, only need to use the calculation program data in a dedicated printer device can print a variety of sophisticated solid model, to solve many of the previous traditional processing can not be solved The problem of all kinds of strange shapes can be $3 \mathrm{D}$ printed out, the design thinking has been emancipated, greatly reducing the production process in a printer, while reducing the processing time, 3D printing is a green manufacturing model, Great savings in the processing of materials[2]. There is no doubt that $3 \mathrm{D}$ printing will change the mode of industrial manufacturing in the past and eventually develop into a new type of manufacturing system[3].

In order to give full play to the advantages of 3D printing, we need to promote the rapid development of 3D printing technology and form an industrial model[4]. First of all, we need to increase innovation in technology, increase investment and achieve important breakthroughs in key core technologies. Second, the government should vigorously support the 3D printing industry and clarify the potential of 3D printing technology. With the help of funds and policies, the government should promote the development of the 3D printing technology industry from a strategic perspective. Third, efforts should be made to expand the application space of 3D printing technology and establish a service platform suitable for 3D printing and processing, giving full play to the advantages of $3 \mathrm{D}$ printing technology[5]. 


\section{D Printing Structure Optimization}

Structural Optimization for Material Saving. 3D printing technology is rapidly evolving, and the cost of 3D printing is declining. Even so, compared with the traditional manufacturing products, 3D printing products and printing costs are still relatively high. Not only 3D printers, 3D printing materials also need a lot of consumption, the current cost of materials generally consumed by the unit volume (Yuan / $\mathrm{cm} 3$ ) to represent. We can conclude that the cost of 3D printing is directly proportional to the volume of material it consumes. Therefore, in order to reduce the cost of 3D printing, we need to optimize the print model to minimize the volume of the model without changing the actual shape and surface quality of the model[6].

How to design a reasonable model to save material? As shown in Fig. 2, For those solid object models, we can adopt the hollowing out method in the middle to calculate the reasonable wall thickness of the product to meet all kinds of strength conditions and hollow out the objects step by step[7]. Due to the various stability issues that can occur after the hollowed-out object, we can add support rods within the appropriate product to reduce both the material and the strength and stability .

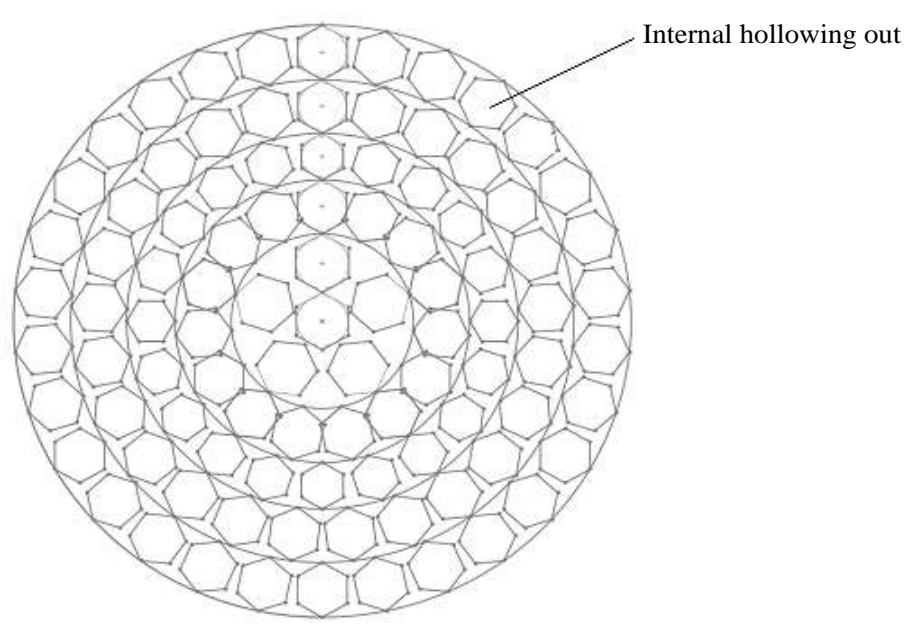

Figure 2. hollowed-out model of the sphere

Structural Optimization for Strength. Everyone can put their ideas into practice, this model helps to develop innovative thinking, but because each person's foundation is not the same, some people do not understand the relevant design experience and physical and mechanical knowledge, Resulting in the design of the product can not be printed out because of a variety of structural problems. Therefore, we need to analyze the intensity of the printed model. When we draw the virtual model on the computer, we should take these problems into account and calculate the position of the structure with insufficient strength, and propose the corresponding improvement measures.

In the process of three-dimensional design, we need to consider the structural areas that are not strong enough, and we need to make a comprehensive analysis of these areas. Considering the influence of the external loads that the structure may exist, We can analyze the mechanics through bending moment, We have to calculate each cross-section assessment, especially those elongated cross-section, it is likely to break because of insufficient strength and fracture. At the same time, we must consider that the printed product may have some deformation, Especially when the printed material is flexible plastic type, We can get the material according to the material properties of the mechanical deformation model, and then on the basis of object deformation as a design parameter to consider the design of elastic object shape. we should compare the deformed model and the model we are looking forward. Integrate all aspects of the factors, so that the product can develop in the direction we look forward. For those three-dimensional models that are calculated and analyzed to 
be under-intensified, we should make measures to correct them, such as thickening at under-strength nodes or adding support rods to the original model[8].

Structural Optimization Oriented to Stability. In actual life, the stability of the object is the balance of the object under the action of various factors. When an object is affected by two or more forces, if the roles of the forces cancel each other, the model will be in Relatively balanced state[9].

In order to solve the problem of printing the stability of the product, we can stick the printed model to the heavy base or make repeated modification to the model until the model can well position. Compared with these two methods, heavy base need to consume a certain amount of material, repeated modifications increase the time cost, The focus optimization method is more reasonable, The focus of the model is adjusted by geometric calculation, so that it can reach a balance state in a particular posture, and maintain enough stability [10].

In order to maintain the stability of objects, we must make the center of gravity of the object in the center of gravity of the object model, The object model is projected to the horizontal axis of the $\mathrm{X}$ axis and the $\mathrm{Y}$ axis, and the polygon formed in the cross section is analyzed so that the center of gravity falls reasonably on the polygon. How to adjust the focus? It means that we can change the focus the model by a certain method so that it reaches a stable equilibrium state. We can hollow out the interior space of the model, so that the center of gravity is adjusted accordingly. Or We can slightly change the shape of the surface of the model and thicken it in some part to offset its center of gravity. After the optimization of the center of gravity, the stability of the model will be significantly improved, without the need for additional support base or support frame, thus it can save both money and time[11].

Support Structure Optimization. Because 3D printing uses a cross-sectional stacking approach to build an object, the planes formed by the $\mathrm{x}$ and $\mathrm{y}$ axes are planes where both the model's cross-sectional profile and its internal fill path lie. When printing a layer, the nozzle moves forward in the z-axis relative to the print support plane by a slice height[12]. Because printed materials are affected by gravity, we need to add support structure below these vacant parts, and wait until the model is printed ,then we should think how to eliminate the support structure.

Therefore, first of all, we need to find the model which need to add support parts, in addition to the model for the corresponding stress analysis, we must also have a basic understanding of the model. For the more horizontal parts, due to the influence of the gravity of the material, the viscosity between the materials is not very good, it is easy to appear the fracture of the section[13]. For the part of the model dangling, the addition of bracing is also necessary. Then it is to ensure that the added support structure can support the corresponding parts. At the same time, the support structure is not the final model we need, and we need to peel the support structure later, so the added support structure should be easy to peel off from the whole and not to damage the surface structure of the model. Then, we also should reduce the number of support rods.

In general, when designing the model of the support structure, we must first consider that the designed support structure needs to have sufficient strength. The extra support rod will increase the printing time, consume more printed materials and increase the cost, At the same time, the shape of the surface of the model will be affected when the support rod is removed later[14]. Nowadays, there are many 3D printing models that print out the support structure all the way from the supported area to the model base. The excessive material consumption causes unnecessary waste. In the three-dimensional drawing software, we can optimize the algorithm, let it automatically generate support structure, and then modify the support structure in the drawing software to reduce the number of supporting structures.

\section{Conclusion}

3D printing is a great innovation in the manufacturing industry. In the future, 3D printing technology will also enter into all aspects of our life just like computers and the Internet. 3D printing has great potential. In order to speed up the pace of $3 \mathrm{D}$ printing, we should actively learn the related knowledge and have a preliminary understanding of the structure. At present, there are still some problems in the optimization of 3D printing model. We must consider the time cost and 
production cost synthetically. We want to optimize the analysis of various algorithms, reduce the time required to print the model, keep the original surface shape of the object model and the physical characteristic. We need to continue to explore the $3 \mathrm{D}$ printing structure to promote $3 \mathrm{D}$ printing technology continues to move forward, so that 3D printing technology can be applied to more and more areas for the general population services.

\section{References}

[1] Y.S. Shi, L.C. Zhang and Y. Bai: Science China Press, Vol. 45 (2015) No 2, p.197

[2] X.J. Zhang, S.Y Tang and H Y Zhao: Journal of Materials Engineering, Vol. 44 (2016) No 2, p. 122

[3] B.X. Shen and Y.P. Guan: Journal of Beijing Information Science \& Technology University, Vol. 31 (2016) No.5, p.60

[4] P. Wang : Distance Education In China, Vol.(2013) No 8, p.83.

[5] X. Li, H Mo and S.S Li: Acta Automatica Sinica, Vol. 42 (2016) No 7, p.983

[6] L.G. Liu, W.P. Xu and W.M Wang: Chinese Journal of Computers, Vol. 38 (2015) No 6, p. 1243

[7] J.Y. Wang and F.M. Dong: Computer Technology and Development, Vol. 26 (2016) No 7, p. 147

[8] X.T. Jiang, X.S. Cheng and N. Dai :Chinese Journal of Mechanical Engineering, Vol. 52 (2016) No 17, p.198

[9] F.F. Wu and L.G. Liu:.Journal of Computer Research and Development, Vol. 54 (2017) No 3, p.549

[10]Musial ski P' Auzinger T and Birsak M: ACM Transactions on Graphics, Vol. 34 (2015) No 4, p. 1021

[11] S.L. Han, Z.Y. Li, Y. Xiao and X. Xu: Machinery Design \& Manufacture, (2015) No.11, p.116

[12] Strano G, Hao L and Everson R: The International Journal of Advanced Manufacturing Technology, Vol. 66 (2013) No 9, p.1247

[13] D.W. Li , N. Dai and X.T. Jiang: Journal of Computer-Aided Design and Computer Graphics, Vol. 28 (2016) No 5, p.841

[14] Y. Chen, S.W. Wang and Z.W. Yang: Science China, Vol. 45 (2015) No 2, p.259 